\title{
Extending the definition of modularity to directed graphs with overlapping communities
}

\author{
V. Nicosia \\ Scuola Superiore di Catania - Laboratorio sui Sistemi Complessi \\ Via S. Nullo 5/i - Catania (ITALY) \\ E-mail: vincenzo.nicosia@ct.infn.it
}

G. Mangioni

Università di Catania - Dipartimento di Ingegneria Informatica e delle Telecomunicazioni - V.le A. Doria 6 - Catania (ITALY)

E-mail: gmangioni@diit.unict.it

\section{Carchiolo}

Università di Catania - Dipartimento di Ingegneria Informatica e delle Telecomunicazioni - V.le A. Doria 6 - Catania (ITALY)

E-mail: car@diit.unict.it

\section{Malgeri}

Università di Catania - Dipartimento di Ingegneria Informatica e delle Telecomunicazioni - V.le A. Doria 6 - Catania (ITALY)

E-mail: mmalgeri@diit.unict.it

\begin{abstract}
Complex networks topologies present interesting and surprising properties, such as community structures, which can be exploited to optimize communication, to find new efficient and context-aware routing algorithms or simply to understand the dynamics and meaning of relationships among nodes. Complex networks are gaining more and more importance as a reference model and are a powerful interpretation tool for many different kinds of natural, biological and social networks, where directed relationships and contextual belonging of nodes to many different communities is a matter of fact. This paper starts from the definition of modularity function, given by M. Newman to evaluate the goodness of network community decompositions, and extends it to the more general case of directed graphs with overlapping community structures. Interesting properties of the proposed extension are discussed, a method for finding overlapping communities is proposed and results of its application to benchmark case-studies are reported. We also propose a new dataset which could be used as a reference benchmark for overlapping community structures identification.
\end{abstract}


Extending the definition of modularity to directed graphs with overlapping communities2

\section{Introduction}

The problem of dividing a graph into "interesting" subgraphs is a classical problem in graph theory. Since graphs are used in many application fields to represent different kinds of structures, from relations among people to connections between computers or interconnections among concepts, there are different reasons and motivations for cutting graphs into smaller components. In the field of computer networks, for example, cutting a network (graph) into smaller components is useful to maximise network bandwidth with respect to access to certain services, while in the case of graphs representing communication between processes an optimal subdivision based on minimizing the flow among group of processes is of the most importance for optimal scheduling in multiprocessors environments.

All those classical problems are often solved by different "clustering methods", which in turn are able to optimize a graph structure in order to guarantee certain desired features, and a huge amount of mathematical literature on that field has been produced.

Nevertheless, studies performed in the last few years on social and natural networks, revealed that algorithms used for graph clustering are neither adept at nor useful for explaining partitioning patterns observed in those networks, such as the arising of "communities", "groups" or "clubs". On the other hand, those kind of structures are really interesting, both for theoretical and for practical reasons. First, because they naturally arise as a consequence of simple interactions among people, and do not require complicated mechanisms to be obtained and maintained. Second, because they have some useful properties, such as high internal connectivity, low path length among nodes and high robustness, which are of the most importance in real applications.

A precise definition of what a "community" really is does not exist yet. One of the most widely accepted and used definitions is that given by Newman and Girvan in [20]: a community is a subgraph containing nodes which are more densely linked to each other than to the rest of the graph or, equivalently, a graph has a community structure if the number of links into any subgraph is higher than the number of links between those subgraphs.

It is not so hard to accept the given definition of communities as a reasonable one: communities in real-life are groups of strongly connected nodes, as happens for example with people in a tennis club, authors in a co-authorship network or colleagues working in the same office [22] [18]. It is worth noting that usually nodes in a community know each other, and the probability for two nodes of a community to have a neighbour in common is higher than for other nodes in the graph [21] 24] [3] [13] [10] [25] [19].

Properties of community structures cannot be revealed by classical algorithms for graph clustering: those algorithms are mainly focused on optimal subdivisions of graphs to guarantee min-flow cuts, while finding communities requires a deeper analysis of

link patterns and relations. For this reason, a significant number of new algorithms for community detection have been proposed in the last few years (refer to [5] [8] for 
Extending the definition of modularity to directed graphs with overlapping communities3

extensive overviews).

Aside from the development of algorithms for community detection, some different metrics for community structure evaluation have been introduced, the most popular and widely accepted of those being the so-called "modularity", defined by Newman [20] [18. Initially defined for undirect networks, the definition of modularity has been subsequently extended to capture community structure in directed networks ([9], [1], [12]).

While an important resolution limit of the modularity measure has been pointed out by Fortunato and Barthelemy in [7], the modularity seems nevertheless to be a useful measure of community structures, and many algorithms for finding graph partitions which give optimal modularity have been proposed already which are able to successfully find communities in really large complex networks[16] [4].

The only drawback of methods based on modularity optimization is that they gives binary partitions of graphs with respect to vertices. In other words, each vertex can be placed into just one community, and no overlaps among communities are allowed. It is still possible to discover sub-communities, iteratively applying those algorithms to each of the partitions found, but discovering partially overlapped communities is not possible at all.

On the other hand, real complex networks are never divided into sharp subnetworks, especially those formed as a result of social relationships and interactions: people usually belong to many different communities, and participate to activities of a certain number of groups at the same time. The problem of discovering overlapping community has been approached in the past in a very few studies ([22], [2], [26], [23], [11]). In this paper we propose to extend the definition of modularity to the more general case of directed graphs with overlapping communities.

The outline of this paper is as follows. In section 2 we give a brief description of the modularity function as defined by Newman. In section 3 we discuss our proposal of modularity suitable for discovering overlapped communities in directed graphs. In section 4 we present a method based on the use of a genetic algorithm to optimize modularity for overlapped communities. In section 5 we show results obtained maximizing the generalized modularity of different complex networks.

\section{Newman's Modularity}

The idea behind Newman's modularity [20] is simple: a subgraph is a community if the number of links among nodes in the subgraph is higher than what would be expected if links were randomly placed. This is exactly what happens in real-world communities, where the number and density of links among people belonging to groups (families, clubs, user groups etc) is higher than expected in a random graph of the same size [15] [21] [24].

This definition of modularity implies the choice of a so-called "null model" [20], i.e. a model of graph to which any other graph can be compared in order to assert the existence of any degree of modularity. When testing for modularity of a complex 
Extending the definition of modularity to directed graphs with overlapping communities4

network, the null model used has so far been a random graph with the same number of nodes, the same number of edges and the same degree distribution as in the original graph, but with links among nodes randomly placed. In such a random graph, the probability $P_{i j}$ of having node $i$ connected to node $j$ is proportional to the degrees (number of links) $k_{i}$ and $k_{j}$ of $i$ and $j$, respectively, and is equal to:

$$
P_{i j}=\frac{k_{i} k_{j}}{4 m^{2}}, \forall i, j
$$

where $m$ is the total number of edges in the graph. If we subdivide an undirected graph $G(E, V)$ into a given number of subgraphs (candidate communities), the modularity of any subgraph $S\left(E^{\prime}, V^{\prime}\right) \subseteq G(E, V)$ with respect to the random-graph null-model can be computed as the sum of differences between the actual number of links among vertices in $V^{\prime}$ and the expected number of links among those nodes in the null-model:

$$
Q_{S}=\sum_{i, j \in V^{\prime}}\left[\frac{A_{i j}}{2 m}-P_{i j}\right]
$$

where $A_{i j}$ are the terms of the adjacency matrix of $G(E, V)$, defined as:

$$
A_{i j}= \begin{cases}1 & \text { if } \mathrm{i} \text { and } \mathrm{j} \text { are connected } \\ 0 & \text { otherwise }\end{cases}
$$

Starting from equation 2, it is possible to define the modularity for the whole graph $G(E, V)$ as follows:

$$
Q=\frac{1}{2 m} \sum_{i, j \in V}\left[A_{i j}-\frac{k_{i} k_{j}}{2 m}\right] \delta\left(c_{i}, c_{j}\right)
$$

where:

$$
\delta\left(c_{i}, c_{j}\right)= \begin{cases}1 & \text { if } \mathrm{i} \text { and } \mathrm{j} \text { belong to the same community } \\ 0 & \text { otherwise }\end{cases}
$$

While this definition of modularity works only for undirected graphs, a straightforward extension to the case of directed graphs has been proposed in [12] [1]. The major change introduced in this extended definition is that the null-model is a directed graph as well, so that $P_{i j}$ is the probability of having a link which starts at node $i$ and ends at node $j$, and conversely $P_{j i}$ is the probability that a link starts at $j$ and ends at $i$. Note that in a directed graph those two probabilities are in general not equal, so the modularity for directed graphs is defined as:

$$
Q_{d}=\sum_{i, j \in V}\left[\frac{A_{i j}}{m}-P_{i, j}\right] \delta\left(c_{i}, c_{j}\right)
$$

where

$$
P_{i j}=\frac{k_{i}^{\text {out }} k_{j}^{i n}}{m^{2}}
$$


Extending the definition of modularity to directed graphs with overlapping communities5

Note that $k_{i}^{\text {out }}$ is the out-degree of node $i$, i.e. the number of links going out of $i$, while $k_{j}^{i n}$ is the in-degree of node $j$, i.e. the number of links coming into $j$.

This formulation of modularity, both for directed and for undirected graphs, has been successfully used to find or confirm community structures of a relatively large number of networks such as the Zachary karate club network[20], the relationship network of dolphins [13], collaboration networks in different research fields [17], and has proved to catch reasonably well the structure of subgroups and communities. The problem with this definition is that communities are sharply separated and it does not take into account possible overlaps among communities in the same network.

\section{Modularity for directed graphs with overlapping communities}

In this section we discuss our proposal to extend the definition of modularity to directed graphs with overlapping communities. We think that this generalization of modularity is needed in order to obtain a metric for evaluation of real-world smooth community structures, such as are emerging from sociological, biological and physical studies recently made in the field of complex networks.

\subsection{Modularity for overlapping communities}

While overlapping among communities is an easy-to-understand concept, since overlapping communities can be found in many real networks, an extension of modularity to evaluate the goodness of overlapped community decomposition is a challenging task. Looking at how modularity was first derived by Newman in [20], the first step is to choose a so-called null-model to be used as a reference for the definition of modularity - As reported in section 2, Newman states that a network is "modular" when the actual number of connections among nodes in a partition is higher than expected for a corresponding random graph, where the random graph is selected as the reference null-model.

In the case of overlapping communities, things are a bit more entangled, because each node can belong to many communities at the same time, and usually it belongs to each community with a certain strength, which is in general not equal for all communities. For this reason, given a directed graph $G(E, V)$ and a set $C$ of overlapped communities built from groups of nodes of $G$, an array of "belonging factors" $\left[\alpha_{i, 1}, \alpha_{i, 2} \ldots \alpha_{i,|C|}\right]$ can be assigned to each node $i$ in the graph, where each coefficient $\alpha_{i, c}$ expresses how strongly node $i$ belongs to community $c$.

Without loss of generality, we can require that

$$
0 \leq \alpha_{i, c} \leq 1 \forall i \in V, \quad \forall c \in C
$$

and that

$$
\sum_{c=1}^{|C|} \alpha_{i, c}=1
$$


Extending the definition of modularity to directed graphs with overlapping communities6

Note that these positions affect just the range of belonging factors, not their meaning: a node can belong to many communities at the same time, with different weights. The strength of each belonging is measured as a real value in the range $[0,1]$ and the sum of all belongings to communities is the same for all nodes in $G$.

Since each node has a belonging coefficient for each community, it is possible to define a coefficient of belonging to each community for edges incoming to or outgoing from a node. We can intuitively suppose that the coefficient of belonging to community $c$ of an edge $l=(i, j)$ which starts at node $i$ and ends at node $j$ can be represented by a certain function of the corresponding belonging coefficients of $i$ and $j$ to community c. In a formula:

$$
\beta_{l, c}=\mathcal{F}\left(\alpha_{i, c}, \alpha_{j, c}\right)
$$

Definition of $\mathcal{F}\left(\alpha_{i, c}, \alpha_{j, c}\right)$ is somewhat arbitrary. It is possible, for example, to define it as the product of the belonging coefficients of the nodes involved, or as $\max \left(\alpha_{i, c}, \alpha_{j, c}\right)$. We actually do not make any choice of a particular form for $\mathcal{F}$.

Even without a precise idea of how a belonging coefficient $\beta_{l, c}$ for a link $l(i, j)$ existing between $i$ and $j$ can be derived from $\alpha_{i, c}$ and $\alpha_{j, c}$, we can still define the nullmodel against which a modularity can be estimated.

Note that it is possible to rewrite equation 5 as:

$$
Q_{d}=\frac{1}{m} \sum_{i, j \in V}\left[A_{i j} \delta\left(c_{i}, c_{j}\right)-\frac{k_{i}^{\text {out }} k_{j}^{\text {in }}}{m} \delta\left(c_{i}, c_{j}\right)\right]
$$

so both the elements $A_{i j}$ of the adjacency matrix and the probability $P_{i j}$ of having a link between $i$ and $j$ in the null model are weighted by the belonging of $i$ and $j$ to the same community, since $\delta\left(c_{i}, c_{j}\right)$ is equal to 1 only when $i$ and $j$ belong to the same community, and it is 0 otherwise. This formulation allows nodes to belong to only one community at a time, and the coefficients which multiply $A_{i j}$ and $P_{i j}$ could just be 0 or 1 , depending on the fact the $i$ and $j$ really belong to the same community.

Things are a bit different if we consider belonging coefficients $\alpha_{i, c}$ as a measure of how much node $i$ belongs to community $c$. In this case, each node can belong to many communities at the same time, and its contribution to the modularity of a given community should be weighted by the corresponding belonging coefficient. We can simply reformulate modularity, where $\delta\left(c_{i}, c_{j}\right)$ is substituted, respectively, by two different coefficients $r_{i j}$ and $s_{i j}$, obtaining:

$$
Q_{o v}=\frac{1}{m} \sum_{i, j \in V}\left[r_{i j} A_{i j}-s_{i j} \frac{k_{i}^{o u t} k_{j}^{i n}}{m}\right]
$$

It is also possible to put in evidence the contribution to modularity given by each community, so that we can rewrite the modularity as:

$$
Q_{o v}=\frac{1}{m} \sum_{c \in C} \sum_{i, j \in V}\left[r_{i j c} A_{i j}-s_{i j c} \frac{k_{i}^{o u t} k_{j}^{i n}}{m}\right]
$$


We can easily derive a convincing formulation for $r_{i j c}$. If community belongings are mutually exclusive (the Newman hypothesis), $r_{i j c}=\delta\left(c_{i}, c_{j}, c\right) \biguplus$ is the portion of the contribute to modularity given by community $c$ due to link $l(i, j)$ and this portion is equal to 1 if and only if $i$ and $j$ are both into the same community and this community is exactly $c$, i.e. if and only if $c_{i}=c_{j}=c$, otherwise it is equal to 0 . If we think of $r_{i j c}$ as the weight of the contribution of $l(i, j)$ to modularity of community $c$, we can define it, in the case of overlapping communities, as the belonging coefficient of $l(i, j)$ for community $c$ :

$$
r_{i j c}=\beta_{l(i, j), c}=\beta_{l, c}=\mathcal{F}\left(\alpha_{i, c}, \alpha_{j, c}\right)
$$

A neat definition of $s_{i j c}$ is a bit more complicated, and requires a clear definition of the null-model to be used as reference. We observed that in graphs which have a significant modularity, the modularity of a partition is measured as the difference between the number of links which are "internal" to each community and the number of total links originated by nodes in the partition. This means that for modular graphs the probability that two nodes belong to the same community is higher if those nodes are neighbours. For this reason, a suitable null-model could be a random graph without a community structure, where the probability for a node to belong to any partition is not related to the fact that any another node belongs to the same partition.

Putting it in a clear way, given a graph $G(E, V)$ we choose as null-model a random graph corresponding to $G(E, V)$ where each node has an out-degree and in-degree as in the original graph, and where no particular community partition can be derived by structural properties of the graph, i.e. where the probability that a node $i$ belongs to a given community $c$ with a belonging factor $\alpha_{i, c}$ does not depend upon the probability that any other node $j$ in the network does belong to the same community with $\alpha_{j, c}$. The latter condition is equivalent to saying that the expected belonging coefficient of any possible link $l(i, j)$ starting from a node into community $c$ is simply the average of all possible coefficients of belonging to $c$ of $l$, so:

$$
\beta_{l(i, j), c}^{\text {out }}=\frac{\sum_{j \in V} \mathcal{F}\left(\alpha_{i, c}, \alpha_{j, c}\right)}{|V|}
$$

Accordingly, the expected belonging coefficient of any link $l(i, j)$ pointing to a node going into community $c$ is:

$$
\beta_{l(i, j), c}^{i n}=\frac{\sum_{i \in V} \mathcal{F}\left(\alpha_{i, c}, \alpha_{j, c}\right)}{|V|}
$$

Those belonging coefficients are used to weight the probability of having, respectively, a link starting at node $i$ and a link pointing to node $j$. Modularity in the case of overlapped communities can be accordingly formulated as:

$$
Q_{o v}=\frac{1}{m} \sum_{c \in C} \sum_{i, j \in V}\left[\beta_{l(i, j), c} A_{i j}-\frac{\beta_{l(i, j), c}^{\text {out }} k_{i}^{\text {out }} \beta_{l(i, j), c}^{\text {in }} k_{j}^{i n}}{m}\right]
$$

$\ddagger$ Note that $\delta c_{i}, c_{j}, c$ is equivalent to $\delta c_{i}, c_{j}$, where we include the community index $c$ only to make it consistent with the formulation of modularity which takes into account contributions given by each community. 
Extending the definition of modularity to directed graphs with overlapping communities8

Note that the extension of the definition of modularity to the case of overlapping communities still depends on the choice of $\mathcal{F}\left(\alpha_{i, c}, \alpha_{j, c}\right)$, i.e. on the way we choose to weight the contribution of each edge to the modularity calculated for community $c$.

The most important properties required for modularity in the formulation given by Newman were that:

(i) $Q=0$ when all nodes belong to the same community, i.e. when no community structure can be inferred from topological considerations

(ii) Higher values of $Q$ indicate stronger community structure

Both of these conditions are satisfied by $Q_{o v}$ as well: if all nodes belong to the same community (condition ili), $|C|=1$ and

$$
\forall i \in V \alpha_{i, 1}=1
$$

as required in order to satisfy equation 8 . At the same time $\mathcal{F}\left(\alpha_{i, c}, \alpha_{j, c}\right)$, which expresses a coefficient of belonging to the unique community $c$ for the edge that links $i$ and $j$, should give a value of 1 for $l(i, j) \forall i, j \in V$, and this condition is fully satisfied, for instance, when $\mathcal{F}\left(\alpha_{i, c}, \alpha_{j, c}\right)$ is a simple average among $\alpha_{i, c}$ and $\alpha_{j, c}$, or if it is the product of the two values or the max between the two. This implies that

$$
\beta_{l, c}^{\text {out }}=\frac{\sum_{j \in V} \mathcal{F}\left(\alpha_{i, c}, \alpha_{j, c}\right)}{|V|}=\frac{|V|}{|V|}=1
$$

and, similarly:

$$
\beta_{l, c}^{i n}=\frac{\sum_{i \in V} \mathcal{F}\left(\alpha_{i, c}, \alpha_{j, c}\right)}{|V|}=\frac{|V|}{|V|}=1
$$

so $s_{i, j}=1$ and the modularity simply reduces to:

$$
Q_{o v}=\frac{1}{m} \sum_{i, j \in V}\left[A_{i, j}-\frac{k_{i}^{\text {out }} k_{j}^{\text {in }}}{m}\right]=0
$$

because

$$
\sum_{i, j \in V} A_{i, j}=m
$$

and

$$
\sum_{i, j \in V} \frac{k_{i}^{\text {out }} k_{j}^{i n}}{m}=m
$$

So condition (ii) is satisfied. At the same time, condition (ii) is satisfied in a network with a modular structure, since the second member of $Q_{o v}$ represents the case of a completely unstructured network, where even the coefficient of belonging to a given community $c$ of a node $i$ is absolutely unrelated with the coefficients of belonging to the same community of nodes in the neighbourhood of $i$. This is clearly not the case in real complex network: it is much more probable that a node's coefficient of belonging to a given community is similar to the coefficients of belonging to the same community of its neighbours. So a strong (overlapping) community structure does imply higher values of $Q_{o v}$, thus satisfying condition (iii). 
Extending the definition of modularity to directed graphs with overlapping communities9

\section{Modularity optimization as a genetic problem}

In the last three decades genetic algorithms (GAs) have played an important role in the field of optimization techniques. Such algorithms are especially suitable when the solution space of a given problem is very large, and an exhaustive search by brute-force for the optimal solution is practically unfeasible.

GAs are mainly based on simulating the creation and evolution of a population of individuals, characterised by a chromosome, where each individual represents a possible solution of the optimization problem. At each simulation step, better individuals are selected, included in a new generation and used to create new elements which replace the worst individuals of the previous generation. The convergence toward a satisfactory solution is obtained applying a set of proper (problem-dependent) operations to each individual. At each iteration, the fitness of all population members is evaluated, and individuals are ordered on the basis of their fitness level. The individuals having the highest fitness value are replicated in the next generation, while new individuals are created, combining the best members of the previous generation using a crossover operation, as well as by performing random mutations.

The most interesting characteristic of GAs is the low computational complexity, while the main drawback is the risk of finding non-optimal solutions, since the optimisation procedure can be stalled by local maxima.

A detailed description of GAs is beyond the scope of this paper. On the other hand we are interested in using GAs for modularity optimization: since $Q_{O V}$ can be considered a fitness function (better decompositions of the graph correspond to higher values of $\left.Q_{o v}\right)$, it is necessary to map the modularity optimization problem into a genetic problem, where the fitness function is $Q_{o v}$ itself and solutions are represented by possible partitions of a graph into overlapping communities. A similar approach for the simple case of maximizing classical modularity has been proposed by Bingol et al. in [14].

\subsection{Chromosome representation}

First of all we need to find a chromosome representation suitable for the given problem. In our implementation the chromosome is represented by a matrix $M=$ $\left(\alpha_{i, c}\right)$, where $i=1, \ldots,|V|$ and $c=1, \ldots,|C|$. Each element $\alpha_{i, c}$ is the strength with which a graph node $i$ belongs to a community $c$. Note that $\alpha_{i, c}$ ranges in the interval $[0.0,1.0]$. Figure 1 shows a graphic representation of the chromosome.

As discussed above, each node of the graph is subject to the following constraint:

$$
\sum_{c=1}^{|C|} \alpha_{i c}=1.0
$$

Equation (19) represents a normalisation to 1.0 of node factors of belonging to the communities. Such a constraint is used to avoid the GA converging toward solutions in which nodes belong to several communities at the same time with a factor 1.0. In other 


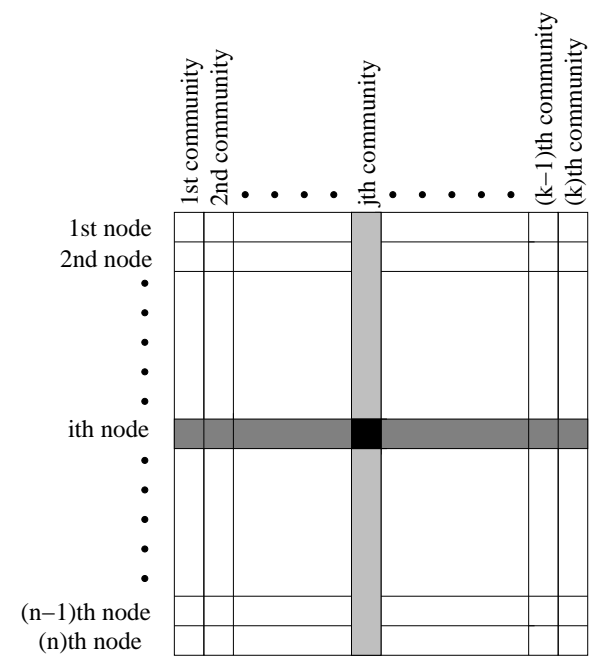

Figure 1. Chromosome representation

words, this means that we are interested in finding solutions for which a node having a unity belonging factor can only belong to one community.

\subsection{The Algorithm}

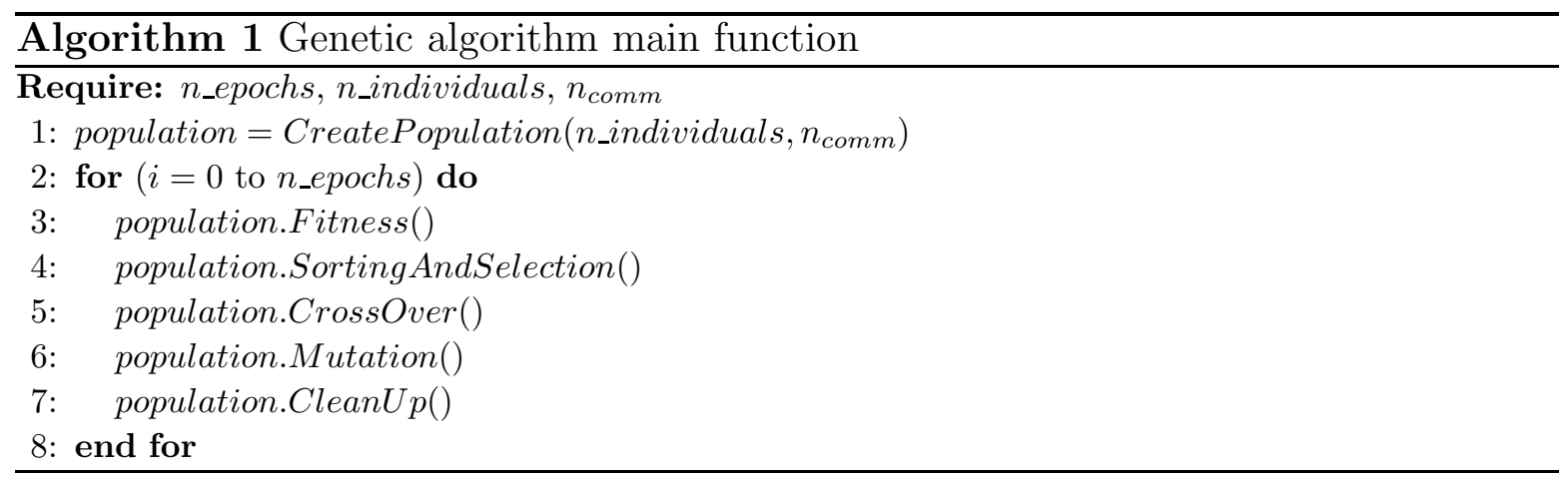

As shown in algorithm1, the algorithm implemented to discover communities takes as inputs three parameters: $n_{-}$epochs, i.e the total number of generations (i.e. the number of simulation cycles); n_individuals, which is the number of individuals making up the population; $n_{\text {comm }}$, the maximum number of overlapped communities that the algorithm will try to find. The last mentioned parameter is not mandatory, but it is useful for reducing the size of the solutions space and to avoid large chromosomes.

The first step of the GA is the creation of the initial population of n_individuals elements. At this step, the chromosome of each individual is initialized with belonging factors chosen at random in the range $[0.0,1.0]$ and normalized in order to respect the constraint expressed by equation (19). After initialization, the GA runs for n_epochs steps, applying at each iteration a set of genetic operators on the population individuals. More specifically, fitness evaluation, selection, crossover and mutations operations are 
performed on the individuals, as explained in detail in the following.

Fitness evaluation, sorting and selection. Fitness evaluation consists in calculating a modularity function for each individuals chromosome. As stated previously, a given number of individuals with higher fitness are included in the next generation. In order to preserve the number of individuals, we need to add new members. They are created as follows: a given number of them is obtained from crossover among better individuals of the previous generation, while the remaining members are created from scratch. In summary, the new generation contains both the best and a combination of better elements of the previous generation.

Crossover and Mutation. Crossover is a genetic operation which consists on exchanging a portion of the chromosomes of two different individuals. The aim of this operation is creating a new individual which inherits its genetic structure (i.e. its chromosome) from the ones of two other population members, hoping that the new individual is better than those it derives from.

In the past, several different crossover techniques have been proposed. In figure 2 the crossover operation that we have used in our GAs is graphically represented. This

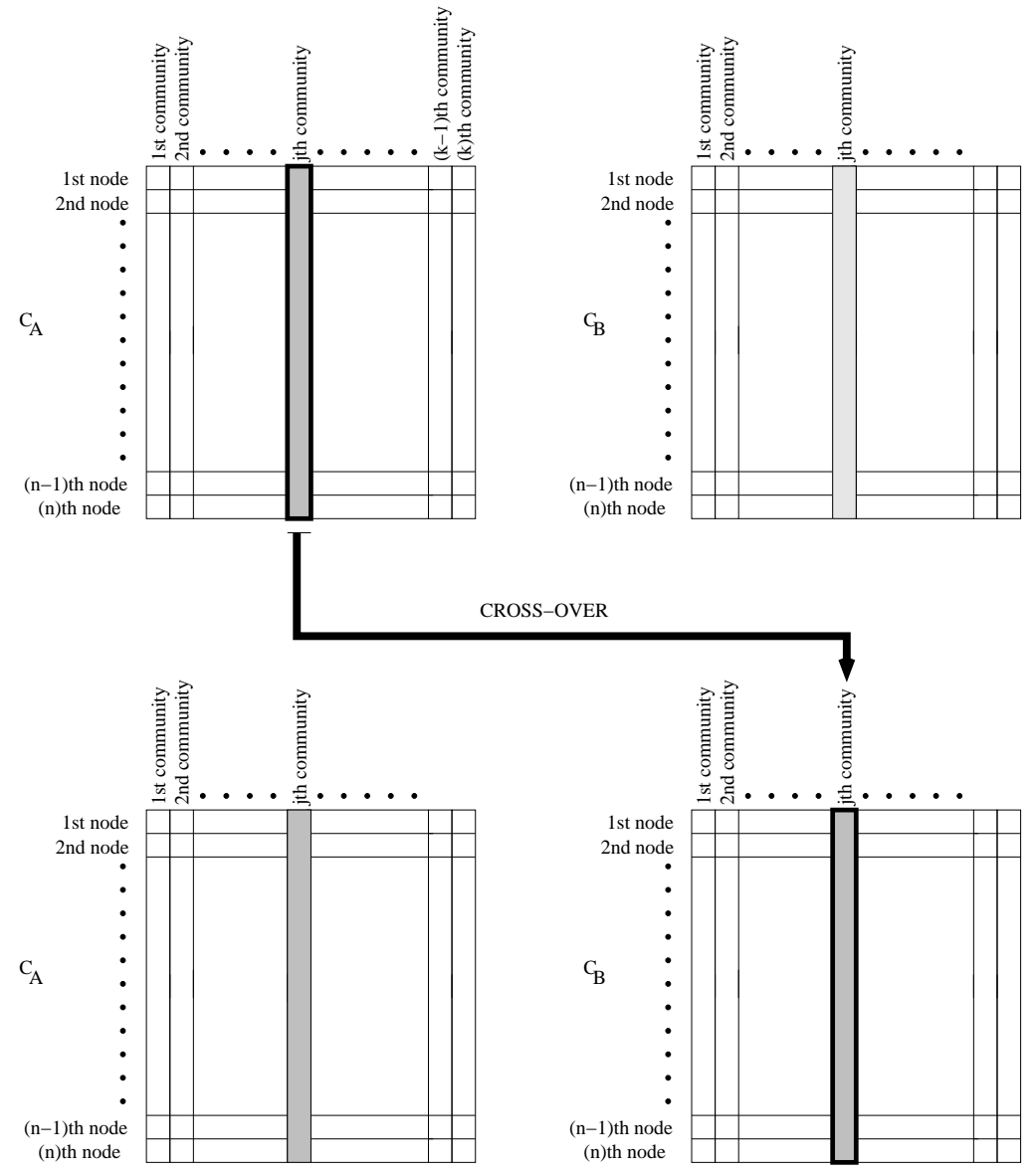

Figure 2. Cross-over operation 
crossover operation takes as inputs chromosomes $C_{A}$ and $C_{B}$, respectively belonging to individuals $A$ and $B$; it works as follows: 1 ) a random number $j$ in range $[1, K]$ is chosen (i.e. a community is randomly selected); 2) the values in column $j$ of $C_{A}$ are copied into column $j$ of $C_{B}$. This crossover defines a one-way operation, since there are values exchanged in one direction only, from $C_{A}$ to $C_{B}$. After a crossover, $C_{B}$ is modified while $C_{A}$ structure remains unchanged.

Moreover, in order to improve the chances of finding the optimal solution, at each iteration mutations are applied on a set of individuals. We have defined a mutation operation that randomly selects a given number of individuals changing in their chromosomes the factor of belonging to a community for a node, where both node and community are chosen at random.

Clean-up. Besides the standard genetic operations, in the proposed GA we have also implemented a CleanUp function with the aim of improving the quality of the graph partition into overlapping communities. Such a function is applied to the chromosome of each individual and it is strictly related to the optimization problem that we are dealing with. In particular, for a given chromosome, a node $i$ and a community $c$ are randomly selected and the average belonging factor avgNeigh $(i, c)$ for community $c$ for neighbours of $i$ is computed (see equation( 201) ). Moreover, the average belonging factor $\operatorname{avgNotNeigh}(i, c)$ for community $c$ of the nodes that are not neighbours of $i$ is evaluated (see equation( 21) ).

$$
\begin{aligned}
& \operatorname{avgNeigh}(i, c)=\frac{\sum_{v \in \text { Neighborhood }(i)} \alpha_{v, c}}{\mid \text { Neighborhood }(i) \mid} \\
& \operatorname{avgNotNeigh}(i, c)=\frac{v \notin N e i g h b o r h o o d(i)}{n-|N e i g h b o r h o o d(i)|} \alpha_{v, c}
\end{aligned}
$$

The values of $\operatorname{avgNeigh}(i, c)$ and $\operatorname{avgNotNeigh}(i, c)$ are then compared: if the former is greater than the latter, the belonging factor of node $i$ for community $c$ is increased by a given small amount; for the contrary, it is decreased. The reason why cleanUp is effective can be explained noting that the neighbour of node $i$ has, on average, a higher probability of belonging to community $c$ than the nodes that are not in neighbourhood of $i$. We make use of this information to drive the algorithm towards a meaningful solution (i.e. towards the optimum) by further increasing the belonging factor of node $i$ for community $k$ to speed up convergence. On the other hand, (i.e. $\operatorname{avgNeigh}(i, c)<\operatorname{avgNotNeigh}(i, c)$ ), decreasing the belonging factor of node $i$ for community $c$ is a way to take into account that node $i$ probably should belong to $c$ less strongly.

In our experiments, we found that the clean-up function significantly improves both the quality of community partitions and the speed of the GA convergence.

After crossover, mutation and clean-up operations, each chromosome could not respect normalization constraint expressed by equation (19). For this reason, a 
normalization operation is performed on each chromosome before starting with a new iteration.

Computational complexity of the algorithm. In order to evaluate the computational complexity of the proposed algorithm, we will only take into account operations depending on the size of the input data, i.e. the number $n$ of network nodes and the number $|C|$ of communities. Analysing the algorithm reported in subsection 4.2, it is possible to note that the most critical operation from the computational complexity point of view is the fitness evaluation. Studying this function, we can conclude that the proposed algorithm has a complexity of $O\left(|C| * n^{2}\right)$ in the worst case.

\section{Results}

In this section we show results obtained maximizing the generalized modularity of different complex networks, and precisely:

- The Zachary Karate Club

- Social relationships of dolphins

- The network of reviews of political books

- The network of students at engineering faculty

The first two networks are well-known to researchers interested in finding community structures, because they have been widely used as benchmarks for testing the quality of new algorithms for community detection. The third network is obtained by connecting together about one hundred political books according to other books that they have been sold together with. Each book has attached a label, corresponding to the political party the author belongs to, according to reviews given by readers. The fourth network is a new dataset built as a complement to this work, obtained by asking students in a computer engineering class at the University of Catania which colleagues they know the most. Following sections describe those networks in depth and report results of $Q_{o v}$ optimization.

As stated in section 3.1, it is necessary to choose $\mathcal{F}\left(\alpha_{i, c}, \alpha_{j, c}\right)$, which expresses the belonging to community $c$ of a link connecting node $i$ to node $j$ as a function of the coefficients of belonging to community $c$ itself of $i$ and $j$.

In our experiments we tried several different bidimensional functions, but the best results so far were obtained so far when $\mathcal{F}$ is a two-dimensional logistic function:

$$
\mathcal{F}\left(\alpha_{i, c}, \alpha_{j, c}\right)=\frac{1}{\left(1+e^{-f\left(\alpha_{i, c}\right)}\right)\left(1+e^{-f\left(\alpha_{j, c}\right)}\right)}
$$

where $f\left(\alpha_{i, c}\right)$ is a simple linear scaling function:

$$
f(x)=2 p x-p, p \in \mathcal{R}
$$


Extending the definition of modularity to directed graphs with overlapping communities 14

Note that $\mathcal{F}\left(\alpha_{i, c}, \alpha_{j, c}\right)$ is practically zero when both $\alpha_{i, c}$ and $\alpha_{j, c}$ are equal to zero, and it is practically 1 when both $\alpha_{i, c}$ and $\alpha_{j, c}$ are equal to 1 , so that condition (ii) is respected. The choice of a two-dimensional logistic function for $\mathcal{F}$ is due to the fact that it is a fairly smooth non-linear version of the $\delta$ distribution used in the traditional

modularity definition, and gives reasonable values for different compositions of $\alpha_{i, c}$ and $\alpha_{j, c} \cdot$

\subsection{The Zachary Karate Club}

The Zachary Karate Club is a social network formed by relationships among people in the same karate club which has been extensively used as a benchmark for all algrithms which aim to discover communities in complex networks. Many algorithms for community detection found the correct partition of this network into the two main communities, as showed in figure 3 .

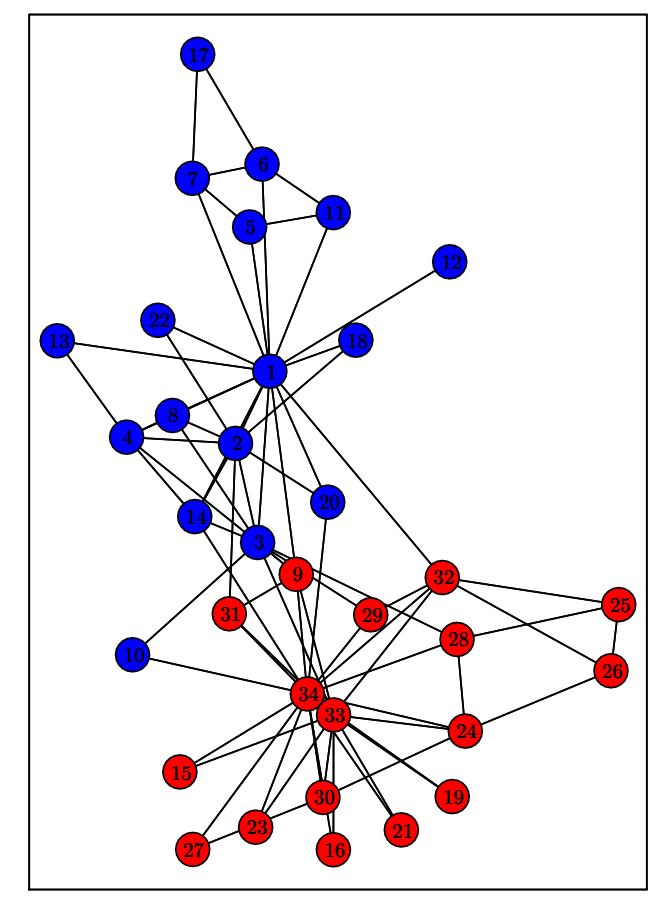

Figure 3. The two main communities of the Zachary Karate Club Network

Nevertheless, further studies on this network showed [6] that if the constraint of having just two communities is relaxed and we search for partitions with more communities, then there is a better decomposition which has a higher modularity value and finds four communities. In particular, the two main communities are further 
divided into two sub-communities; the real decomposition of the Zachary Karate Club is depicted in figure 4 .

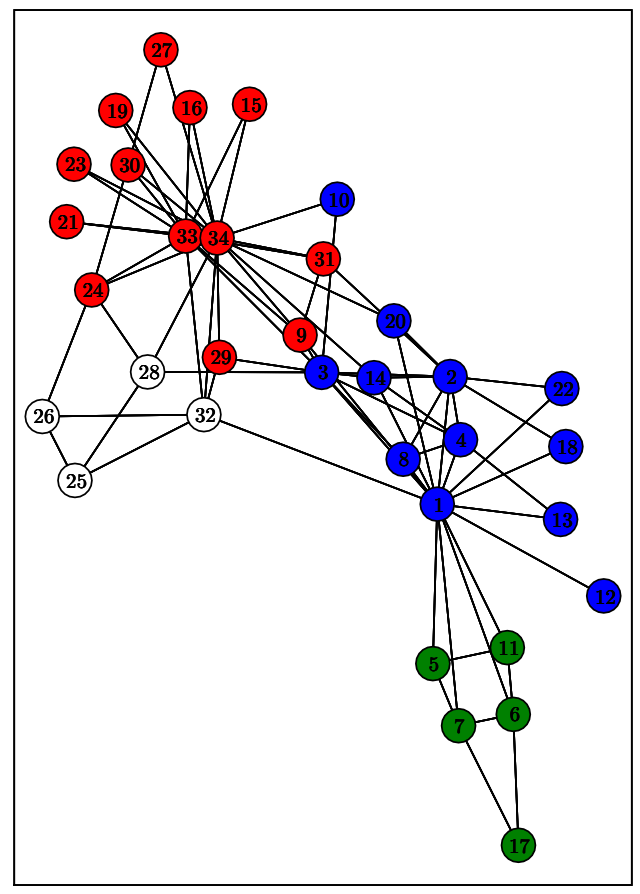

Figure 4. The four communities found maximising the modularity of the Zachary Karate Club Network

Using GAs for optimization of $Q_{o v}$ for the Zachary network, and fixing the number of communities to be found (as mentioned in section 4.2) at 2, then the best decomposition obtained by the GA finds the maximum modularity when $\alpha_{3,0}=0.81$ and $\alpha_{10,0}=0.63$, while $\alpha_{i, 0}=1.0$ or $\alpha_{i, 0}=0.0 \forall i \notin[3,10]$, according to the correct placement of nodes into the community in which they belong.

The overlap among the two communities is shown in figure 5. The two lateral lines indicate community boundaries: all the nodes which are to the left of the leftmost vertical line belong only to the first community, and all nodes to the right of the rightmost vertical line belong only to the second community. The vertical line in the middle of the graph corresponds to a perfect overlap, i.e. if a node stands on that line than it belongs $50 \%$ to the first community and $50 \%$ to the second.

While the solution reported in figure 5 is found by the GA in $98 \%$ of the runs, a couple of other solutions which have almost the same modularity value sometimes come out as local maxima. These solutions discover a couple more nodes as overlapped among communities, even if the belonging coefficients for their original community are really close to 1 . These solutions are interesting in pointing out that it is important to 


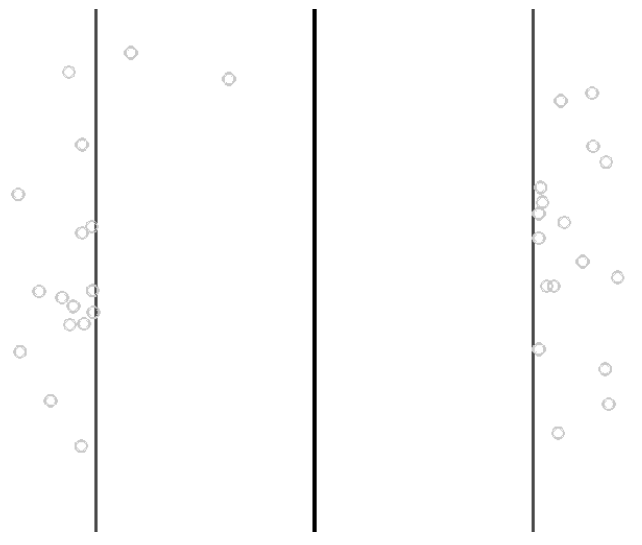

Figure 5. Overlap among the two communities in the Zachary Karate Club - optimal solution

take into account not only the partition which has "the best" modularity, but also other partitions which are sub-optimal but still meaningful.

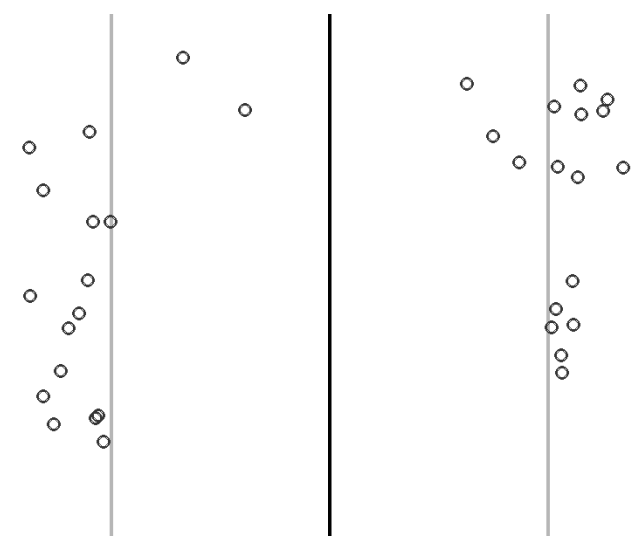

Figure 6. Overlap among the two communities in the Zachary Karate Club - suboptimal solution

In figure [ one of these sub-optimal solution is reported. See how the best solution is somehow included in this one, since node 3 and 10 are overlapped with almost the same belonging coefficients as in the best case, while other overlaps appear in the other communities: those nodes are nodes 9, 31 and 34, and all of them are in the border of their community, and have links to nodes in the first community as well.

After having constrained the GA to explore the space of solutions in the case of a partition with only two communities, we tried to maximise modularity raising the upper bound of communities to be found by the GA to ten. The surprising result was that six of the ten available communities were left completely empty by the algorithm, and just four of them contain nodes: two of them correspond to the two original communities found by all algorithms, with the overlaps of node 3 and 10, while the other two communities are the two sub-communities found in [6] massively overlapped with the main communities. 
This result clearly shows that the generalised formulation of modularity is not only able to capture overlaps but, to some extent, also to take into account hierarchical organisation of communities.

\subsection{The social network of dolphins}

Nodes in a social networks do not necessarily have to be humans, and links among nodes in such networks are not limited to friendship. An extension of the concept of social network is possible also for groups of animals, where links usually express some kind of relationships among them, e.g. the fact that they belong to the same family or that they have been observed together. One of the most famous social network of animals was deeply described in [13] and is a network of social relationships among dolphins: two dolphins are connected if they have been seen swimming together.

The (perhaps not so) surprising property of the resulting network is that at least two major communities exist: the first one is formed by females, while the other contains only males individuals. Nevertheless, it has been discovered that both those communities are further divided into sub-communities, and the resulting picture is shown in figure 7.

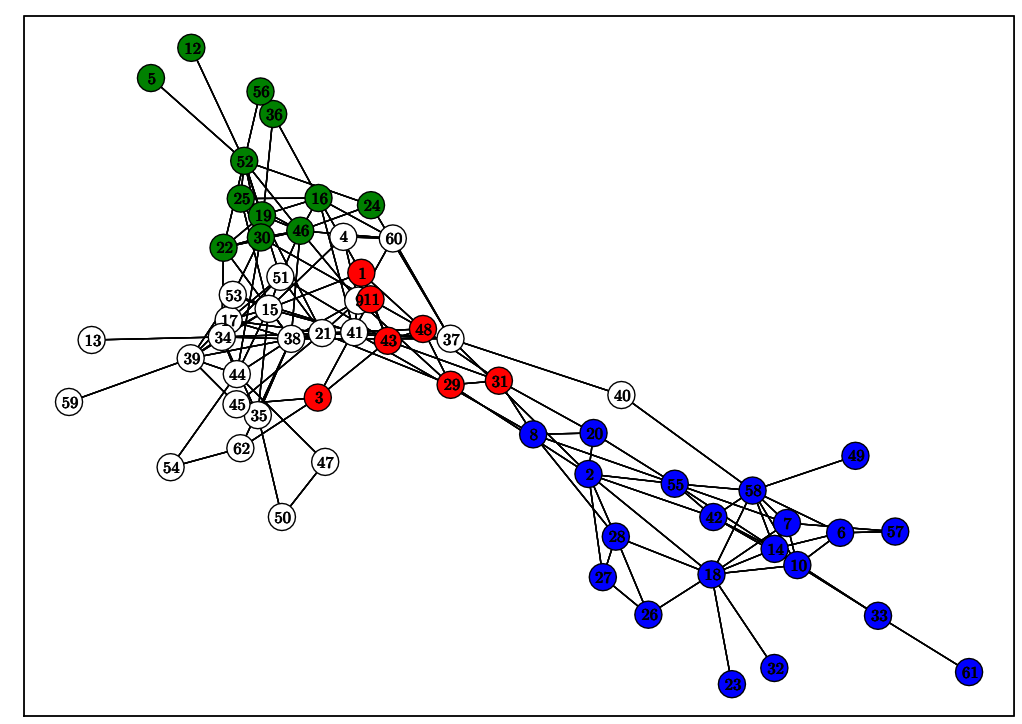

Figure 7. The four sharp communities found in the dolphins network

White and green nodes represent females, while red and blue are for males. Note that all females are correctly grouped together as are males, and that subcommunities (i.e. green and red nodes) lie somewhere in the middle between the two main communities (i.e. white and blue nodes). 


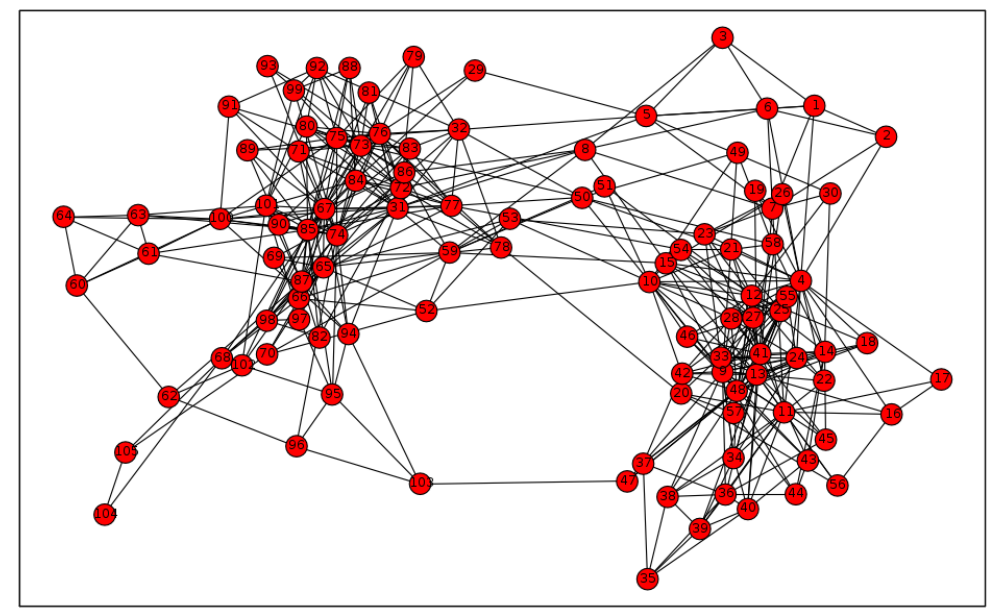

Figure 8. The polbooks network

The results obtained maximizing the generalized modularity of the dolphins network are similar to those observed for the Zachary Karate Club: the four communities are successfully found, and a slight overlap among sub-communities of each major community is found. For example, almost all nodes in the small red sub-community of males have a small overlap with the blue community, and the same happens for nodes in the green sub-community of females.

\subsection{Political Books}

The problem of the two networks examined so far is that no information about existing overlaps among communities is available. In the case of the Zachary network, it is unfeasible to recover such information since it would be very difficult to find all the people involved in the experiment and ask them if the overlap found for nodes 3 and 10 is meaningful, or whether the two sub-communities discovered really were strong subgroups. In the case of dolphins network, it is simply impossible to state which overlap makes sense, and which sub-communities have any real meaning, since this network is based on correlation of observations made, and not on what animals have ever said about their real relationships!

On the other hand, information about overlaps in groups and communities is not given for the majority of networks, and should be inferred by other characteristics of each network.

A complex network which indirectly gives information about overlaps among communities is the so-called "PolBooks" network (see figure 8. This network is an information network where each node represents a political book sold by Amazon (most of them have been published and edited in the U.S.) and a link between two nodes exists if a book has been purchased in combination with another book on the same topic.

Additionally, each node in the network has been marked as "liberal", "conservative" or "neutral", according to the reviews written by readers. We expect that this network 
Extending the definition of modularity to directed graphs with overlapping communities19

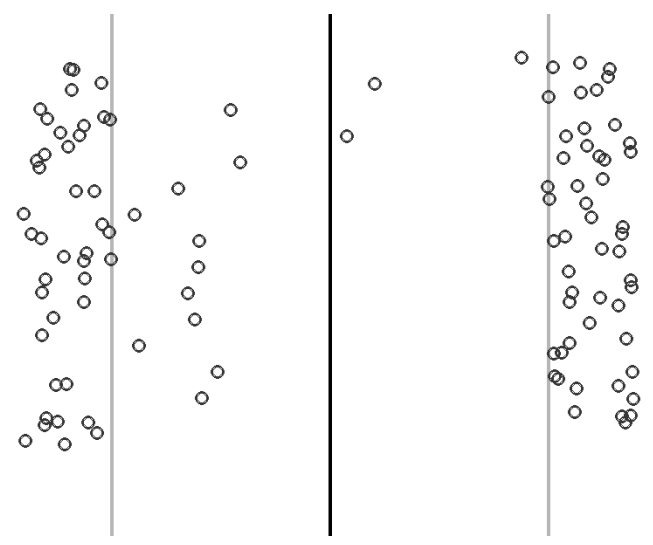

Figure 9. Optimal partition for the polbooks network

would be naturally divided into three communities, but the majority of algorithms for community detection find the best partition with two or four communities, where nodes labelled as "neutral" are often put into the "conservative" or "liberal" party group.

Another image emerges if we maximize the generalized modularity of the PolBooks network: the best decomposition is that with two overlapped communities, where conservative and liberal books represent the larger communities and neutral books are placed as overlapped among the two, with a small number of books labelled as liberal also overlapped with the conservative community. Figure 9 shows this. Note that all the conservative books (nodes on the right) form a strong community while a modest number of liberal books show an overlap between the two communities. All books labelled as neutral are in the middle, and confirm that in such networks the best decomposition into communities is not sharp and precise, and that overlaps among communities catch the internal organisation of the network better.

\subsection{Students in computer engineering}

The last example given in this section is a novel network, built with the precise objective of testing generalised modularity on graphs which are intrinsically divided into overlapping communities.

The network is composed by students of computer engineering course at the University of Catania: all students attend the third year, and they are divided into two class, depending on the first letter of their last name, so that all students whose last name starts with a letter from $\mathrm{A}$ to $\mathrm{L}$ is in the first class, while other students are in the second class. Each class has the same academic curriculum, but attends lessons with different teachers, and no common courses are attended by all students together, mainly because of space limitations.

Students were asked, through an anonymous test, which one of their colleagues they knew the best and considered as friends, both in their own class and in the other one. Each student was assigned a unique ID, so that other students should use just IDs 
to answer the question, in order to preserve the privacy of students. The only personal information known is the first letter of the name corresponding to each ID, so that it is possible to label each student as belonging to the first or to the second class.

It is easy to imagine that the network obtained by connecting nodes which relate to friends should have communities, since it is much easier for students to have friends in their own class than in the other, and the two communities should be almost sharply separated, since no academic overlap exists among the two groups.

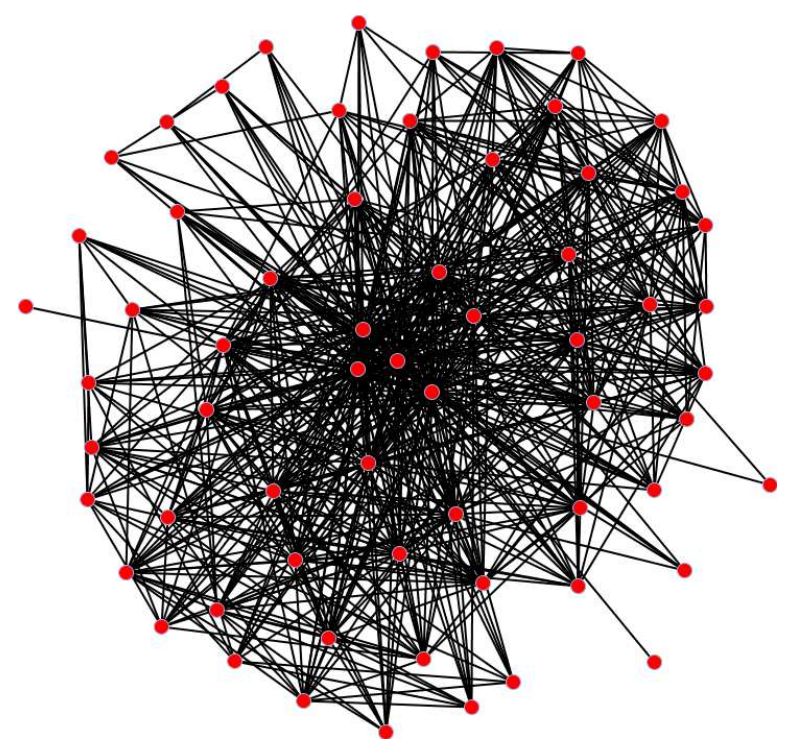

Figure 10. Engineering students network

Nevertheless, a really surprising result came out of the experiment: the resulting network (see figure 10), which has about sixty nodes and more than one hundred and fifty links, is quite well connected, and a really low average path length has been observed. On the other hand, the network has a clear community structure, but the best decomposition is obtained when about one-third of the nodes are overlapped among the two communities. This result is shown in figure 11 .

The fact that two groups of students with no common academic activities can have such a massive overlap sounds strange and somewhat unusual. We tried to find possible causes of this overlap, but nothing seemed to can explain it in a crystal clear way.

Suddenly, we remembered what could seem a secondary detail: all the students involved in the experiment, who where attending the third year courses divided into two classes, had been previously divided into three classes during their first year at university, each class containing students whose name started with a letter from A to $\mathrm{F}$, from $\mathrm{G}$ to $\mathrm{P}$, and from $\mathrm{Q}$ to $\mathrm{Z}$, because they were too many to fit in just two classes! This is the explanation of the mysterious overlap: the two communities of students were originally three communities and, when the community in the middle was separated and absorbed by the two final communities, some of the relationships among students put in two different classes survived, so the resulting network naturally has communities 
Extending the definition of modularity to directed graphs with overlapping communities 21

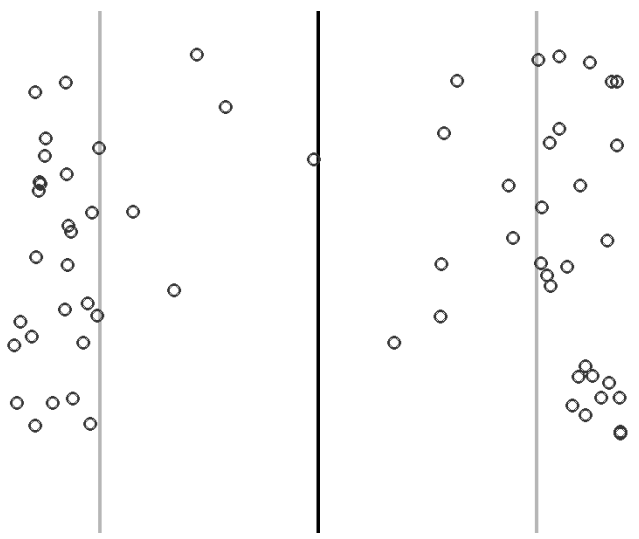

Figure 11. Overlaps among the two communities in the engineering students network

with massive overlaps. Optimization of generalized modularity rediscovered a forgotten property of the network, finding once again the best solution.

The network of students could be used, in the future, as a reference benchmark for testing capabilities of algorithms for detection of overlapping communities in complex networks.

\section{Conclusions}

This paper proposed an extension of modularity function for directed graphs with overlapping communities. This generalization moves from simple considerations about the meaning and structure of the original modularity function, using an enriched nullmodel which takes into account nodes belonging to more than just one community at the same time. The function used to estimate the contribute of an edge to the modularity of a community does not affect the formal procedure used to derive the generalized modularity, and has been left apart for a future research. Moreover, a method for overlapping communities discovery based on the use of a genetic algorithm for the optimization of the extended modularity function is presented. Finally, we discuss results of the application of our proposal to several complex networks.

\section{Acknowledgments}

The authors gratefully acknowledge Santo Fortunato for a careful reading of the manuscript and for helpful suggestions.

\section{References}

[1] A. Arenas, J. Duch, A. Fernandez, and S. Gomez. Size reduction of complex networks preserving modularity. New Journal of Physics, 9:176, 2007. 
Extending the definition of modularity to directed graphs with overlapping communities22

[2] J. Baumes, M. Goldberg, and M. Magdon-Ismail. Efficient identification of overlapping communities. Lecture notes in Computer Science. IEEE International Conference on Intelligence and Security Informatics (ISI 2005), 3495:27-36, 2005.

[3] Markus Brede and Sitabhra Sinha. Assortative mixing by degree makes a network more unstable. arXiv:cond-mat/0507710v1, 2005.

[4] Aaron Clauset, M. E. J. Newman, and Cristopher Moore. Finding community structure in very large networks. Physical Review E, 70:066111, 2004.

[5] Leon Danon, Jordi Duch, Albert Diaz-Guilera, and Alex Arenas. Comparing community structure identification. Journal of Statistical Mechanics: Theory and Experiment, P09008, 2005.

[6] J. Duch and A. Arenas. Community detection in complex networks using extremal optimization. Physical Review E, 72:027104, 2005.

[7] Santo Fortunato and Marc Barthelemy. Resolution limit in community detection. PROC.NATL.ACAD.SCI.USA, 104:36, 2007.

[8] Santo Fortunato and Claudio Castellano. Community Structure in Graphs. Encyclopedia of Complexity and System Science. Springer, 2008.

[9] R. Guimera, M. Sales-Pardo, and L. A. N. Amaral. Module identification in bipartite networks with applications to directed networks. Physical Review E, 76:036102, 2007.

[10] Petter Holme. Network dynamics of ongoing social relationships. Europhysics Letters, 64:427, 2003.

[11] Andrea Lancichinetti, Santo Fortunato, and Janos Kertesz. Detecting the overlapping and hierarchical community structure of complex networks. New journal of Physics, 11(033015), 2009.

[12] E. A. Leicht and M. E. J. Newman. Community structure in directed networks. Physical Review Letter, 100:118703, 2008.

[13] David Lusseau and M. E. J. Newman. Identifying the role that individual animals play in their social network. PROC.R.SOC.LONDON B, 271:S477, 2004.

[14] Tasgin M., Herdagdelen A., and Haluk Bingol. Community detection in complex networks using genetic algorithms. arXiv:0711.0491, 2007.

[15] M. E. J. Newman. The structure and function of complex networks. SIAM Review, 45:167, 2003.

[16] M. E. J. Newman. Fast algorithm for detecting community structure in networks. Phys. Rev. E, 69(6):066133, Jun 2004.

[17] M. E. J. Newman. Finding community structure in networks using the eigenvectors of matrices. Physical Review E, 74:036104, 2006.

[18] M. E. J. Newman. Modularity and community structure in networks. PROC.NATL.ACAD.SCI.USA, 103:8577, 2006.

[19] M. E. J. Newman and M. Girvan. Mixing patterns and community structure in networks, pages 66-87. Springer, Berlin, 2003.

[20] M. E. J. Newman and M. Girvan. Finding and evaluating community structure in networks. Physical Review E, 69:026113, 2004.

[21] M. E. J. Newman and Juyong Park. Why social networks are different from other types of networks. Physical Review E, 68:036122, 2003.

[22] Gergely Palla, Imre Derenyi, Illes Farkas, and Tamas Vicsek. Uncovering the overlapping community structure of complex networks in nature and society. Nature, 435:814, 2005.

[23] Gergely Palla, Illes J. Farkas, Peter Pollner, Imre Derenyi, and Tamas Vicsek. Directed network modules. New Journal of Physics, 9:186, 2007.

[24] Juyong Park and M. E. J. Newman. The origin of degree correlations in the internet and other networks. Physical Review E, 68:026112, 2003.

[25] R. Xulvi-Brunet, W. Pietsch, and I. M. Sokolov. Correlations in scale-free networks: Tomography and percolation. Physical Review E, 68:036119, 2003.

[26] S. Zhang, R.-S. Wang, and X.-S. Zhang. Identification of overlapping community structure in complex networks using fuzzy c-means clustering. Physica A, 374:483-490, 2007. 